\title{
2014. AÑO DEL CABALLO DE LA ECONOMÍA MUNDIAL
}

\author{
2014 YEAR OF THE HORSE THE WORLD ECONOMY \\ Beatriz Herrera García ${ }^{*}$ \\ Docente Principal de la Facultad de Ciencias Contables \\ Universidad Nacional Mayor de San Marcos-UNMSM / Lima-Perú \\ [Recepción: Setiembre de 2014/ Conformidad: Octubre 2014]
}

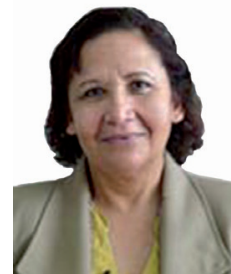

\section{RESUMEN}

La economía mundial mejorará en el presente año, aunque mantiene riesgos de vulnerabilidad.

Concretamente, se estima según el Proyecto LINKy el Modelo del DAES, un crecimiento del tres por ciento (3.0\%) a escala global, tasa superior al $2.1 \%$ alcanzado durante el 2013.

En el 2014, se espera que los países en desarrollo continúen siendo los impulsores del crecimiento económico mundial y se contempla; asimismo, que las políticas adoptadas en los Estados Unidos y el Japón contribuyan a que estas economías repunten y también, favorezcan un mayor crecimiento económico en el mundo. Asimismo, en la zona del euro (UE), según pronósticos recientes, un aumento del crédito, políticas fiscales menos restrictivas y un repunte de las exportaciones contribuirían al logro de tasas de crecimiento positivas. Sin embargo, se espera que el crecimiento en la zona del euro se mantendrá en un nivel bajo durante un prolongado tiempo. Pero una recuperación sólida de la economía norteamericana y el retorno de China a un crecimiento sostenible facilitarían la recuperación en la UE.

\section{Palabras clave:}

Grave recesión; economías desarrolladas; políticas restrictivas; políticas expansivas.

\begin{abstract}
The world economy will improve this year, while maintaining vulnerability risks.

Specifically, it is estimated according to the LINK Project and Model DESA, an increase of three percent (3.0\%) on a global scale, higher rate reached $2.1 \%$ in 2013.

In 2014, it is expected that developing countries continue to be the drivers of global economic growth and is contemplated; also that the policies adopted in the United States and Japan contribute to these economies to rebound and also promote further economic growth in the world. Also in the euro area (EU), according to recent forecasts, an increase in credit, less restrictive fiscal policies and a rebound in exports contribute to achieving positive growth rates. However, it is expected that growth in the euro area will remain at a low level for a long time. But a strong recovery of the US economy and China's return to sustainable growth facilitate recovery in the EU.
\end{abstract}

\section{Keywords:}

Serious recession; developed economies; restrictive policies; expansionary policies.

* Doctora en Ciencias Económicas. Email : bherrerag@unmsm.edu.pe 


\section{INTRODUCCIÓN}

En el presente artículo se asume la finalización de la severa recesión que afectaba, principalmente, a los países desarrollados. En la zona del euro ${ }^{1}$, que era afectada adversamente por el comercio y los flujos financieros globales; así como, la elevada volatilidad e incertidumbre de mercados y consumidores, en medio de dificultades para adoptar políticas que permitan corregir desequilibrios y el rápido deterioro de la actividad económica es causa y efecto de la crisis de la deuda soberana y de los problemas fiscales en dichas economías.

La deuda del sector público en varias economías europeas aumentó en el 2012 y agravó la debilidad de los balances de los bancos. Las drásticas medidas adoptadas por los gobiernos para lograr una reestructuración ordenada de la deuda de Grecia generaron simultáneamente turbulencias financieras y crecientes preocupaciones de riesgo de moratoria de pagos en algunas de las mayores economías de la zona, en particular Italia.

Durante el 2013, en la zona del euro el escaso dinamismo del crédito privado, una tasa de desempleo que se encumbró en niveles máximos históricos (en especial, en España, Portugal y Grecia), una persistente incertidumbre y una política fiscal contractiva fueron parte de la severa y prolongada recesión.

Por otro lado, desde la crisis, los flujos internacionales de capital privado hacia los países en desarrollo se han mantenido extremadamente volátiles, en parte asociados a los temores de los inversionistas de cartera respecto a la sustentabilidad de las finanzas públicas en Europa, generando una huida hacia inversiones seguras. Asimismo, muchos bancos europeos enfrentaban presiones hacia un mayor desapalancamiento en sus balances, lo que se tradujo en reducciones de los préstamos hacia países en desarrollo. La desaceleración en Brasil, China e India redujeron los flujos hacia estos países.

En cambio, la coyuntura mundial en el 2014 señala un punto de inflexión de la severa recesión debido fundamentalmente a:
- Según los recientes pronósticos, la crisis económica de la zona del euro entra en una fase de terminación. La dinámica compuesta por la crisis de la deuda soberana y la fragilidad del sector bancario, deja de afectar las hojas de balance tanto de los gobiernos como de los bancos comerciales, como resultado de los acuerdos de políticas para brindar asistencia a los miembros que lo requieran. Además, un aumento del crédito, políticas fiscales menos restrictivas y un repunte de las exportaciones contribuirán a que la zona del euro alcance tasas de crecimiento positivas.

- En los Estados Unidos, se ha llevado a cabo una política de estímulos, estableciendo como meta alcanzar una tasa de desempleo del 6.5 por ciento para retirar el programa (de estímulos monetarios). A mediados del año pasado la Fed ${ }^{2}$, anunció el posible fin del programa (de expansión monetaria) a mediados de 2014. El anuncio generó la caída de las principales bolsas del mundo e importantes ventas en los mercados, especialmente, de renta fija, así como, la ampliación de la prima de riesgo de las deudas soberanas de una gran parte de los países del mundo. Ello, sugiere que ante el retiro de los estímulos monetarios por parte de la Fed, la volatilidad se hará presente en los mercados del dinero y de acciones, lo que plantea un serio desafío a las autoridades monetarias del mundo entero.

- Asimismo, el gobierno norteamericano superó la amenaza del llamado "abismo fiscal", al alcanzar acuerdos políticos con las partes. Por otro lado, no se ha producido una brusca desaceleración en los principales países en desarrollo, incluyendo a China. Brasil, China e India tuvieron periodos de fuerte crecimiento previo a la crisis financiera. Ambos aspectos han influido positivamente ante las incertidumbres acerca de la demanda externa y los riesgos de una fuerte desaceleración en el crecimiento, que no se produjo en las tres grandes economías mencionadas.

\footnotetext{
1 Se denomina zona del euro a los países de la Unión Europea (EU-15): Austria, Bélgica, Dinamarca, Finlandia, Francia, Alemania, Grecia, Irlanda, Italia, Luxemburgo, Holanda, Portugal, España, Suecia e Inglaterra.

2 Reserva Federal de los Estados Unidos de América (la Fed).
} 
- En Japón la banca central decidió en abril de 2013, reorientar su política hacia una de estímulos monetarios, con el objetivo de reactivar la economía japonesa, teniendo como meta una inflación del 2.0 por ciento, que podría tener resultados positivos a corto plazo. La recuperación de las exportaciones japonesas, favorecida por la depreciación del yen y cierto crecimiento de la producción manufacturera son signos de reactivación.

- China se encuentra en un periodo de transición para orientar su economía hacia el consumo, tendencia que hasta el 2013 no ha podido consolidarse, los recientes resultados de la economía china han sido inferiores a las expectativas de los analistas. Es de recordar, que China ante una reducción de sus exportaciones, el crecimiento de la inversión contribuyó en más del 50 por ciento al crecimiento del producto bruto interno (PIB) en las últimas décadas ${ }^{3}$. Sin embargo, esta contribución puede ser afectada por las políticas más restrictivas en el mercado de la vivienda, mayor precaución en las medidas de estímulo fiscal y restricciones al financiamiento hacia los gobiernos locales para la implementación de nuevos proyectos. Asimismo, China tiene amplio espacio en sus políticas para rebalancear su economía hacia un mayor peso de la demanda doméstica, incluyendo un mayor gasto del gobierno central en servicios públicos como salud, educación y seguridad.

- En consecuencia, se asume, que la nueva situación financiera mundial se destacará por la continuación de las políticas monetarias expansivas entre los países desarrollados, lo que favorecerá la recuperación del crecimiento mundial de tasas débiles a tasas positivas moderadas. En opinión de los analistas de la Cepal y; en especial, de su Secretaría Ejecutiva, se debe tener en claro que la actual situación corresponde a un ciclo eco- nómico distinto al de la expansión que se tuvo entre el 2003 y el 2008, ahora corresponde a otra etapa del ciclo donde habrá menos dinamismo del consumo y del comercio. En el 2013, Europa estuvo en niveles de crecimiento negativo, en el 2014 se estima que no, porque ha comenzado a recuperarse lentamente. La economía de Estados Unidos, se ha vuelto a dinamizar, eso va permitir que China vuelva a ser también, una economía más dinámica. El consumo en China va a tomar un papel más relevante, antes era la inversión ${ }^{4}$.

\section{LA COYUNTURA MUNDIAL EN 2014}

Durante el 2013 la economía mundial se había recuperado, pero manteniendo un perfil continuo moderado, lo que permite considerar que habrá signos alentadores durante el 2014, los indicadores económicos de la zona del euro fueron; finalmente, moviéndose más allá de su prolongada recesión para cambiar a un crecimiento lento, tanto en los Estados Unidos y el Japón (la recesión mundial provino de estas economías desarrolladas). Sin embargo, el comercio mundial ${ }^{5}$ sigue siendo inferior a los niveles previos a la crisis, pero como la demanda se estimuló ante la mayor confianza, el comercio ganará impulso en los países desarrollados; así como, en los países en desarrollo.

Por otro lado, hay un riesgo en desarrollo, se trata de la volatilidad de los flujos, en particular con respecto a la disminución gradual del programa de flexibilización cuantitativa por la Fed, que tuvo consecuencias fuertes. El potencial de riesgos a la baja sustancial de la disminución gradual y prematura del programa de flexibilización cuantitativa, podría afectar al crecimiento de la economía mundial.

La mayoría de los analistas económicos espera que la economía mundial crezca a un ritmo del 3.0

\footnotetext{
3 Debido a ello, existen importantes riesgos de un crecimiento significativamente menor. Si, por ejemplo, su crecimiento se redujera en cerca del 5.0 por ciento anual (causado por una mayor desaceleración de la inversión, mayores restricciones al mercado de la vivienda y ausencia de nuevos estímulos fiscales), Los países en desarrollo, en conjunto, podrían sufrir una pérdida acumulada del PIB de cerca del 3.0 por ciento durante el periodo 2013 - 2015, y el mundo una pérdida del producto mundial del 1.5 por ciento.

4 En opinión de Alicia Bárcena, Secretaria Ejecutiva de la Comisión Económica para América Latina (Cepal), En China hay una fuerte migración del campo a la ciudad que va cambiar el patrón de consumo, eso quiere decir, que habrá mayor demanda en el área alimentaria.

5 El comercio mundial enfrentaba una notable desaceleración debido a la recesión de la zona del euro y de Estados Unidos. El débil crecimiento registrado fue de: 12.6 por ciento en 2010; 6.4 por ciento en 2011 y 3.2 por ciento en 2012 .
} 
por ciento en el 2014. Asimismo, se espera que el producto bruto interno de los Estados Unidos al 2.5 por ciento en el 2014, (véase, cuadro $\mathrm{N}^{\circ} 1$ ), mientras que se espera que Europa Occidental crezca un 1.5 por ciento en el presente año, debido a que está saliendo de la prolongada recesión. Es de recodar que un número creciente de países desarrollados europeos habían recaído en recesión, mientras que otros agobiados por los problemas de deuda soberana la habían profundizado, es así, que muchos países desarrollados se encontraban atrapados en una espiral negativa caracterizada por el alto desempleo y una débil demanda agregada, compuesta por la austeridad fiscal, elevada carga de deuda pública y fragilidad financiera.
En cambio, las políticas expansionistas de Japón están demostrando su eficacia, aunque las próximas reformas estructurales no generan la misma confianza. El crecimiento de la economía de Japón se espera que crezca un 1.5 por ciento en el 2014.

En China, se espera que el crecimiento pueda mantener un ritmo de alrededor del 7.5 por ciento (véase, cuadro $\mathrm{N}^{\circ} 1$ ). Mientras que la economía de la India, se espera que crezca en 5.3 por ciento en el 2014. Las expectativas de crecimiento para Brasil y Rusia son más modestas, 3.0 por ciento y 2.9 por ciento, respectivamente.

\section{Cuadro No 1}

Pronósticos del crecimiento en las principales economías del mundo*

\begin{tabular}{|l|c|c|}
\hline & $\mathbf{2 0 1 4}$ & $\mathbf{2 0 1 5}$ \\
\hline Mundo & 3.0 & 3.3 \\
\hline Unión Europea & 1.4 & 1.8 \\
\hline Estados Unidos & 2.5 & 3.2 \\
\hline Japón & 1.5 & 1.2 \\
\hline China & 7.5 & 7.3 \\
\hline India & 5.3 & 5.7 \\
\hline Brasil & 3.0 & 4.2 \\
\hline Rusia, Federación & 2.9 & 3.6 \\
\hline
\end{tabular}

Fuente: UN/DESA Report: World Economic Situation and Prospects.

*Según el Proyecto LINK. (Es un grupo de investigación de colaboración Internacional para la creación de modelos econométricos, coordinado conjuntamente por la División de Análisis y Políticas de Desarrollo de UN/DESA y la

Universidad de Toronto).

Según de los resultados de los pronósticos registrados en el Cuadro $\mathrm{N}^{\circ} 1$, y del Informe del Departamento de Asuntos Económicos y Sociales de Naciones Unidas, se espera que en los Estados Unidos de América y países de la zona del euro ${ }^{6}$ el fin de la prolongada recesión o por lo menos que la misma ceda positivamente durante el presente año. En cambio, se estima que China e India experimenten un moderado crecimiento.
En general, como se observa en el Cuadro $\mathrm{N}^{\circ} 1$, el crecimiento del producto bruto mundial ha tenido un comportamiento similar al de los periodos 2012 y 2013, antes de recuperarse gradualmente y lograr, según los pronósticos, una tasa de $3.0 \%$ en el 2014, con tendencia creciente hacia el 2015. Es decir, se mantiene en una trayectoria creciente: $2.2 \%$ en el 2012 a $2.4 \%$ en el 2013 y se espera lograr 3.0 por ciento en 2014. Sin embargo, esta moderada trayec- 
toria de crecimiento económico no será capaz de recuperar la fuerte pérdida de empleo causada por la severa depresión.

La mejora esperada para el 2014, se basa en las mejores condiciones financieras globales y la reducción de riesgos a corto plazo. La economía mundial sigue expandible a un ritmo moderado, después de un descenso marcado en los últimos años, por lo que se predice que la actividad económica mundial gane poco a poco impulso durante el año en curso. Es probable, también, que la mayoría de las regiones del mundo vean un fortalecimiento moderado. Sin embargo, es de advertir que el crecimiento se mantendrá por debajo del potencial.

\section{ECONOMÍAS DE MERCADO DESARROLLADAS. PERSPECTIVAS}

La economía mundial ha experimentado un crecimiento moderado durante el año anterior, siendo un resultado incapaz de satisfacer las modestas proyecciones de muchos analistas. Durante el 2013 la economía mundial experimentó una zaga en casi todas las regiones del mundo debido a que siguió enfrentado "vientos en contra". Un bajo rendimiento en la economía, se observó en casi todas las regiones y los principales grupos económicos. La mayoría de las economías desarrolladas siguieron luchando contra los efectos persistentes de la crisis financiera, luchando a favor de los retos de la adopción de medidas de política fiscal y monetaria apropiadas.

Sin embargo, algunos signos de mejora han aparecido recientemente: la zona del euro; finalmente, ha salido de su prolongada recesión, con el producto interno bruto de la región de vuelta al crecimiento. La economía de los Estados Unidos, se estima crecerá a un ritmo magro pero positivo, debido al endurecimiento fiscal y a una serie de "cuellos de botella" políticos de orden presupuestaria que han pesado mucho en el crecimiento. Su política monetaria que ha sido acomodaticia ha tenido un mayor efecto en el aumento de los precios de las acciones que en el estímulo de la economía real.
El conjunto de estos nuevos elementos fundamentan un mejor pronóstico acerca del producto bruto mundial, basados en esos elementos nuevos y varios supuestos, se prevé que la economía mundial crezca a un ritmo del $3.0 \%$ en el 2014 y $3.3 \%$ en el 2015 (véase, cuadro $\mathrm{N}^{\circ} 1$ ). Este pronóstico de base se hace en un contexto de una serie de incertidumbres y riesgos que surgen de los posibles errores de política y de factores que escapan al ámbito económico. A pesar de las diferencias notables en las tasas de crecimiento entre los distintos grupos de países, los movimientos cíclicos en el crecimiento permanecen sincronizados.

Como se señaló anteriormente, en el caso norteamericano, las expectativas que surgieron a mediados del año pasado, ante una posible disminución gradual del programa de flexibilización cuantitativa, causaron nerviosismo en los mercados financieros, elevando las tasas de interés a largo plazo. Según el Informe WEO del FMI, entre los riesgos de los mercados financieros cabe mencionar, las tasas de interés a largo plazo inesperadamente altas en Estados Unidos y la posibilidad de que se revierta la reciente tendencia a la baja de las primas de riesgo y la volatilidad. En el panorama económico, en el supuesto de que el futuro desenrollado de la relajación monetaria será suave, se espera que el PIB de los Estados Unidos aumente 2.5 y $3.2 \%$ para los años 2014 y 2015 , respectivamente ${ }^{7}$.

Europa ha salido de la recesión a duras penas según sus autoridades, ellos afirman "Salimos de la recesión en el segundo semestre de 2013, impulsado por las exportaciones netas y, en menor medida, por el consumo privado y público, pero la inversión se mantuvo débil y el desempleo se sitúo en alza", por lo que se espera que el PIB crezca 1.4 y 1.8 por ciento en los años 2014 y 2015, respectivamente. El crecimiento seguirá siendo débil por una serie de factores como: los programas de reducción en la intensidad de una carga en la demanda intrarregional siga siendo excepcionalmente baja, y la demanda extraregional se mantenga ralentizada. Las condiciones del crédito siguen siendo restrictivas para algunos países; en particular, para las pequeñas y medianas empresas (PYMEs). Una diversidad considerable se 7 En el supuesto que el techo de la deuda sea levantado y el futuro desenrollado de la relajación monetaria será lisa. Sin embargo, persisten
los riesgos a la baja, sobre todo por la disputa política sobre el presupuesto. 
encuentran entre los países de la UE, por ejemplo: Reino Unido y Gran Bretaña e Irlanda del Norte con un crecimiento relativamente fuerte, seguido de Alemania, y por otro lado, los países en crisis se mantienen en posiciones muy débiles, con Chipre, Grecia y Portugal, que se espera permanezcan en recesión durante el 2014.

\section{A. Estados Unidos. Hay mejora en las perspectivas de crecimiento, pero los riesgos a la baja siguen siendo altos}

Como se afirmaba (supra), la mayoría de las economías de mercado desarrolladas, por fin han entrado en un periodo de crecimiento después de más de cinco (5) años con los efectos de la crisis financiera global. Una variedad de políticas han promovido efectivamente el crecimiento y la estabilidad en estas economías.

En las economías de mercado desarrolladas las perspectivas mejoran, por ejemplo, la economía de los Estados Unidos ha resistido los "vientos en contra" de las medidas fiscales generadas que ayudaron, en cierta medida, como la compra a gran escala de activos a largo plazo por la Fed. Simultáneamente, nuevas y audaces políticas de estímulo de Japón, han trabajado para impulsar el crecimiento y la deflación final en esa economía. En la zona del euro; así como, en el resto de Europa Occidental, la salida de la recesión fue debido a las políticas del Banco Central Europeo (BCE) implementadas para la estabilización de la confianza en la región. Sin embargo, la actividad económica en la región sigue siendo débil en los países más desarrollados, debido a que persiste un alto desempleo.

En los Estados Unidos, la mejora de las perspectivas de crecimiento se enfrenta al endurecimiento fiscal y a una serie de "cuellos de botella" políticos sobre cuestiones presupuestarias. Las incertidumbres sobre el techo de la deuda y el presupuesto siguen vigentes. Si bien, la política monetaria ha sido acomodaticia, los tipos de interés a largo plazo comenzaron a aumentar en el segundo semestre de 2013, debido a las preocupaciones sobre la disminución gradual del programa (conocido como flexibilización cuantitativa o de expansión monetaria-QE).
Por otro lado, se espera que el gasto de los consumidores se fortalezca moderadamente, por lo que el consumo privado crecerá ligeramente en el 2014 debido a dos factores de apoyo que incluyen efectos sobre la riqueza: la recuperación de los precios de la vivienda y el aumento de los precios de las acciones. Además, el modesto aumento de la renta disponible generada por el lento pero continuo crecimiento del empleo, han contribuido positivamente al incremento del consumo privado. En el lado negativo, la confianza del consumidor ha sido frecuentemente perturbada por las incertidumbres asociadas a las luchas políticas en torno a las cuestiones fiscales. El endurecimiento fiscal, incluyendo impuestos sobre la renta más altos, aplicados al vencimiento del alivio en los impuestos sobre las nóminas y la incertidumbre sobre el gastos del gobierno, han puesto freno negativamente al gasto de consumo. Por su parte, muchas familias siguen emprendiendo el desapalancamiento financiero, al reducir los préstamos hipotecarios en relación con sus ingresos. Sin embargo, durante el periodo de previsión, se espera que algunos de los efectos adversos disminuyan y el gasto de los consumidores se expanda en un 2.5 y un 2.7 por ciento en los años 2014 y 2015, respectivamente.

La inversión empresarial ha experimentado una marcada desaceleración el año anterior, con un crecimiento de 2.4 por ciento, menor que el 7.3 por ciento de crecimiento alcanzado en el 2012, debido a las incertidumbres asociadas a la política fiscal que han retrasado las decisiones empresariales en los gastos de capital y de la planificación de proyectos, pero se espera que el ritmo de crecimiento de la inversión fija se retraiga levemente en los años 2014 y 2015.

La inflación ha sido benigna, con el índice de precios al consumidor (IPC), el aumento de la inflación de una tasa promedio de $1.5 \%$ en el 2013 , se considera controlable por lo que se espera permanezca por debajo del $2.0 \%$ en el periodo 2014 y 2015.

Por el lado del comercio exterior las exportaciones reales de bienes se desaceleraron notablemente durante el 2013, creciendo alrededor de 2.0 por ciento, por debajo del 3.8 por ciento del año anterior. Las exportaciones de bienes y equipos informáticos disminuyeron, pero las exportaciones de aeronaves 
y otros bienes de consumo aumentaron sólidamente. Las importaciones reales de bienes crecieron moderadamente los dos últimos años, a tasas de 2.2 y 1.7 por ciento. Las importaciones de productos derivados del petróleo así como, el de computadoras crecieron a un ritmo similar, por lo que se espera aumenten alrededor del 5.0 al 6.0 por ciento en el periodo $2014 \mathrm{y}$ 2015. Con todo, se estima que el déficit comercial sea alrededor de 420 mil millones de dólares en el 2013. Asimismo, se supone que tanto el déficit comercial $y$ el déficit de cuenta corriente permanezcan en sus ratios con relación al PIB en los años 2014 y 2015.

En materia de política monetaria como se dijo anteriormente, la Fed ha mantenido una política monetaria muy acomodaticia durante el 2013, aplicando dos instrumentos: mantener la tasa de interés de los fondos federales al cero y el aumento de las compras de bonos del Estado a largo plazo y valores respaldados por garantías hipotecarias. Así, el rango objetivo con garantía hipotecaria se mantendrá; así como, la tasa de fondos federales en niveles excepcionalmente bajos, siempre y cuando la tasa de desempleo se mantenga por encima de 6.5 por ciento, o la inflación se sitúe en niveles de los dos años anteriores. Por lo que se proyecta aumente en no más de la mitad de un punto porcentual por encima del 2.0 por ciento del PIB, a largo plazo. Por lo tanto, se asume que la tasa de interés de los fondos federales se mantenga dentro del rango de 0.0 a 0.25 por ciento hasta mediados de 2015. Asimismo, se asume que la Fed reduzca gradualmente las cantidades de sus compras en el 2014.

La política fiscal de los Estados Unidos se ha reforzado durante el 2013 a través de dos canales: la reducción de dos puntos porcentuales en los impuestos de nómina y un aumento de impuestos a la renta, para el uno por ciento superior de los hogares de altos ingresos, y la activación de los recortes de gastos automáticos en todos los ámbitos por un valor de 85 mil millones de dólares en el 2013. El resultado debe ser, un gasto público en términos reales, con una disminución en alrededor del 5.0 por ciento. Sin embargo, durante el periodo de pronóstico 2014-2015, se espera que la política fiscal siga siendo restrictiva, pero menos severa que el 2013. En fin, se espera que el gasto público sea plana (lisa).
Con todo, se puede afirmar que los principales riesgos para la economía de los Estados Unidos están asociados tanto con la política monetaria y la política fiscal. La Fed se enfrenta a un dilema: la compra de activos a largo plazo por demasiado tiempo podría causar burbujas de activos, pero si acorta demasiado el tiempo podría ahogar la recuperación económica y desestabilizar los mercados financieros. El riesgo relacionado con la política fiscal puede ser aún más grave, ya que la división política en el parlamento continúa en relación al techo de la deuda y las cuestiones presupuestarias.

\section{B. Japón sale de la deflación pero la elevada deuda pública sigue siendo un problema}

Japón con paquetes de política económica reactivadores logró la aceleración de la economía y la recuperación. Un conjunto nuevo de audaces medidas de políticas de estímulo adoptadas a finales de 2012, ha impulsado el crecimiento económico en Japón y puso fin al periodo de deflación, que duró una década.

El producto interno bruto (PIB) de Japón se estima que crecerá un 1.9 por ciento en el 2013, y la variación anual del índice de precios al consumidor (IPC) se ha movido de valores negativos a valores levemente positivos. Sin embargo, el déficit presupuestario del gobierno central sigue siendo importante; así como, el de la deuda pública, que es el más alto entre todos los países desarrollados, considerándolo como porcentaje del PIB; con el riesgo de que siga aumentando.

En adelante, se espera que el gobierno introduzca otra serie de políticas dirigidas a las reformas estructurales, junto a la implementación del aumento previsto de las tasas de impuestos al consumo en los próximos dos años. Mientras, los efectos de las reformas estructurales previstas sigan siendo inciertas, las mayores tasas de impuestos al consumo pueden frenar la demanda agregada.

En adelante, el crecimiento del producto interno bruto del Japón se proyecta a tasas moderadas (véase, cuadro $\mathrm{N}^{\circ} 1$, supra), los estimados son de 1.5 por ciento y de 1.2 por ciento en el periodo 2014-2015, respectivamente. 
La política fiscal japonesa aún no está haciendo hincapié en la austeridad. El estímulo fiscal incluyó un presupuesto suplementario de 10.3 trillones de yenes para el año fiscal que terminó en marzo de 2013.

Se espera, en el periodo de pronóstico, que la tasa de impuestos sobre el consumo se incrementará desde el actual nivel del 5.0 por ciento al 8.0 por ciento en abril de 2014, y al 10.0 por ciento en octubre de 2015. A finales del presente año, el gobierno introducirá otro estímulo presupuestario de alrededor de 5 trillones de yenes, para compensar los efectos negativos de los impuestos más altos. El déficit fiscal en el 2014 será similar al 10.0 por ciento del PIB alcanzado el año anterior.

En relación a la política monetaria Japón ha alterado la dinámica de precios, así el Banco de Japón (BoJ), anunció su nueva política de relajación monetaria cuantitativa y cualitativa (QQme) el 4 de abril de 2013, que iba dirigida a una duplicación de la base monetaria en dos años a través de la compra de bonos del gobierno japonés (JGB) y de los demás instrumentos financieros, a montos de 60-70 billones de yenes por año. El alcance de la compra de bonos también se amplió para incluir JGB de largo vencimiento. El BoJ espera reducir los rendimientos de los valores a largo plazo para aumentar las expectativas de inflación de los consumidores, las empresas y los inversionistas. El objetivo final es aumentar la tasa de inflación anual del IPC al 2.0 por ciento en dos años.

El impacto temprano de la QQme en los rendimientos de los bonos japoneses ha sido notable y parece ser sostenible. La QQme también ha tenido un impacto significativo sobre el yen japonés, que se depreció vis-avis con el dólar norteamericano en un 21 por ciento anual, a partir de octubre de 2013.

Japón había experimentado un largo periodo de deflación, durante 15 años consecutivos desde 1998. Por lo que, con la QQme han cambiado las expectativas de inflación de los agentes económicos, así lo revelan las encuestas realizadas a mediados de 2013. La fuerte depreciación del yen japonés, también ha aumentado la presión hacia el alza de los precios de los bienes importados.

Las decisiones de políticas están modificando la dinámica del consumo y la inversión. Durante el año anterior, el consumo privado aumentó 1.8 por ciento, apoyado por la confianza del consumidor fortalecido $y$, en parte, por el adelanto de las compras de bienes duraderos para evitar el impuesto al consumo más alto. Correspondientemente, el crecimiento del consumo privado se desacelerará a alrededor de uno por ciento en los últimos años. Asimismo, durante el año anterior, la inversión fija recibió un gran impulso por el continuo crecimiento de los proyectos de obras públicas financiadas por el presupuesto suplementario, también la inversión residencial creció en respuesta al impuesto sobre el consumo más alto. Se espera que la inversión crecerá 2.0 por ciento y 1.3 por ciento en 2014 y 2015, respectivamente.

\section{Europa. En las economías europeas las tensio- nes en la región han disminuido}

En las economías de Europa Occidental la recesión ha terminado pero el crecimiento sigue siendo débil. Así, las autoridades económicas afirman "Salimos de la recesión en el segundo trimestre de 2013, después de seis (6) trimestres consecutivos de descenso del PIB”. Se espera que la actividad económica seguirá expandiéndose pero a un ritmo débil.

Las tasas de crecimiento anual se verán afectadas negativamente por la fuerte recesión de finales del 2012 y principios del 2013, por lo que el PIB de la UE-15 (véase pie de página, supra), disminuyó en $0.1 \%$ en el 2013 , se espera luego se fortalezca a tasas de $1.4 \%$ y $1.8 \%$ en los años 2014 y 2015 , respectivamente. La débil pero continua recuperación de la "Gran Recesión” ha causado que el nivel del producto de la Unión Europea (UE), se sitúe 2.8 por ciento por debajo del potencial. Sin embargo, hay diferencias considerables entre países. Entre los países mayores, se espera que el Reino Unido de Gran Bretaña e Irlanda del Norte crezcan $1.4 \%$ en el 2013 y se fortalezcan al 2.2 por ciento en 2014, mientras se espera que Francia y Alemania crezcan 0.1 por ciento y 0.4 por ciento en el mismo periodo, respectivamente, para luego expandirse hasta el 0.8 por ciento y 1.9 por ciento en el 2014. Los países en crisis que se mantienen en posiciones delicadas pero que están mostrando poder dar la vuelta de la esquina, como Italia, se espera se contraiga 1.8 por ciento en el 2013 antes de salir de la recesión, para luego crecer en 
0.8 por ciento en el 2014, del mismo modo, se estima que España se contraiga 1.2 por ciento en 2013 antes de volver a un crecimiento positivo de 0.9 por ciento en el 2014. Entre los países en crisis más pequeños, se asume por problemas de deuda principalmente que Chipre y Grecia sigan contrayéndose en el 2014.

Las tensiones en la región desarrollada de Europa han disminuido drásticamente desde que el Banco Central Europeo (BCE) anunciara las facilidades de sus transacciones monetarias simples (OMT). A pesar de que la política aún no se ha activado, los diferenciales de los bonos soberanos se han reducido significativamente, y las crisis pequeñas que se produjeron a principios de 2013 casi ninguna produjeron reacciones en el mercado de bonos. No obstante, la región enfrenta "vientos en contra" de cara al futuro, tales como: los programas de austeridad fiscal, aunque en menos intensidad permanecerán vigentes; la demanda intrarregional continúa siendo excepcionalmente baja, mientras que la demanda extrarregional se ha ralentizado. La reestructuración de los balances sigue siendo un proceso en curso para los bancos, las sociedades no financieras y los hogares. Las condiciones de los préstamos son heterogéneos: con el crédito bancario ampliamente disponible en algunos países, y en otros, sigue siendo extremadamente muy condicionado.

Sin embargo, es de advertir que la fuerte desaceleración de la inflación a finales de 2013, conlleva algunos riesgos de deflación en 2014.

El consumo se está recuperando pero sigue enfrentando "vientos en contra”. Los gastos de consumo siguen siendo débiles y se estima que ha disminuido ligeramente en la zona del euro en los dos últimos años, sin embargo, se observa que se ha frenado por una serie de factores: el desapalancamiento de los hogares, lo cual es un legado permanente de la "Gran Recesión”; el general mal estado de los mercados del trabajo; la baja confianza del consumidor, que se ha visto muy afectado después de cada etapa de la crisis de la zona euro; los programas de austeridad fiscal, $y$ los altos precios de la energía, que han deprimido los ingresos reales. En adelante, se espera que el gasto de consumo se recupere modestamente, ya que muchos de los factores mencionados han disminuido en intensidad, o en algunos casos la confianza ha vuelto. En particular, el consumo se ha estabilizado y aumentado constantemente desde que el alivio de las tensiones en la región ha cesado. Comola intensidad de los programas de austeridad y el estado de los mercados del trabajo han variado enormemente en toda la región, lo que ayuda a explicar en cierta medida el robusto gasto en consumo en Austria, Francia y Alemania.

El gasto en inversión ha sido y sigue siendo un punto débil, cayó bruscamente en la mayoría de los países en el periodo 2012 y 2013, y se espera un rebrote débil en el futuro. La débil demanda, la incertidumbre continua, el desapalancamiento y las dificultades de financiación en los países en crisis han sido las restricciones clave, por lo que los préstamos comerciales a las sociedades no financieras seguirán contrayéndose. Se espera que la inversión aumente en 2014 y 2015, así como la demanda se recupere poco a poco, pero el rebrote será débil. La utilización de la capacidad productiva ha aumentado desde comienzos del año, pero sigue siendo baja en relación a los estándares históricos.

Las condiciones de financiación varían enormemente en toda la región, las tasas de interés de los préstamos, en particular, a las empresas pequeñas y medianas (PYMEs), son mucho mayores en los países en crisis, que en el resto de la región; esta situación durará algún tiempo para normalizarse.

El crecimiento del volumen de las exportaciones sigue siendo bajo en la zona del euro, los volúmenes de exportación crecieron sólo 1.2 por ciento en 2013, como consecuencia de la extrema debilidad de la demanda intrarregional, junto a la ralentización de la demanda extrarregional (en particular, de la proveniente de Asia Oriental). La depreciación del euro durante el año pasado ha "humedecido" aún más las exportaciones.

Como la demanda regional y global han disminuido, las exportaciones tienden a hacer lo mismo, con el apoyo de la depreciación del euro (asumido durante el periodo de pronóstico), los volúmenes de importación serán aún más débiles, en el 2013 han disminuido por segundo año consecutivo. Sin 
embargo, algún rebrote se observa para los años 2014 y 2015, debido a que el crecimiento regional mejorará. Aunque la depreciación del euro podría afectar negativamente a las importaciones, la evolución de la demanda será el factor dominante.

\section{CONCLUSIONES}

1. Se están dando un conjunto de factores que indican que el crecimiento económico mundial está fortaleciéndose en el presente año. Estos son: las economías de la zona del euro han salido de su prolongada recesión; las economías de Estados Unidos y Japón se vienen fortaleciéndose; China e India consiguieron contrarrestar la desaceleración que sufrieron en los dos últimos años y están evolucionando favorablemente aunque de forma moderada.

2. A partir de 2011, las principales economías desarrolladas de América del Norte, Europa y Asia están alineadas en una trayectoria ascendente de lento crecimiento, lo que hace suponer constituya una recuperación de la recesión, luego de cinco años de permanecer atrapadas en una serie crisis financiera. Las tasas de crecimiento pronosticadas no son suficientes para recuperar la producción y las pérdidas de empleo generadas por la crisis.

3. Las economías de la zona del euro, teóricamente, salieron de la grave recesión en el segundo semestre de 2013. En el conjunto de los 15 Estados miembro de la Unión Europea, se espera un aumento del producto bruto interno en el periodo 2014-2015. Sin embargo, la recuperación sigue siendo débil, debido a que el PBI sigue estando por debajo del nivel que tenía antes de la crisis financiera. En este escenario de falta de dinamismo en la región, sumada al perfil de crecimiento lento, de monedas fuertes y precios más bajos de los productos básicos, está dando lugar a tasas extraordinariamente bajas de inflación, generando temores de un cuadro de deflación. La debilidad de la recuperación influye considerablemente en la grave situación del desempleo (en las economías de la zona del euro llegaba al $11.1 \%$ en 2013). Esta cifra esconde las grandes disparidades existentes en la región debido al desigual grado de desarrollo, por ejemplo en Alemania la tasa de desempleo es de 5.5 por ciento, en cambio en España dicha tasa llega al 25.9 por ciento. Teniendo en cuenta las tasas altas de desempleo, la inflación exhibe niveles extremadamente bajos (en la zona del euro la inflación se sitúa por debajo del uno por ciento), esta situación aunada a la persistente debilidad del crecimiento de la producción, reavive los temores de una deflación similar a la de Japón difícil de revertir.

4. En los nuevos Estados miembros de la Unión Europea la recuperación se viene dando en condiciones que dichas economías siguen funcionando por debajo de su potencial y la inversión directa extranjera resulta decepcionante. Sin embargo, se espera que las inversiones en general, deberían aumentar aprovechando el aumento de la confianza empresarial y la absorción de los fondos de la UE.

5. Para la economía de Japón se proyecta una expansión del 1.5 por ciento para el año en curso debido al paquete de estímulo fiscal que se introdujo en el 2013. Una política monetaria poco convencional implementada a inicios de 2013, hizo bajar la rentabilidad de los valores a largo plazo orientando las expectativas de inflación hacia un nivel más alto. También, dio lugar a una considerable depreciación del yen japonés con respecto a otras divisas duras, produciendo un nuevo aumento de la inflación. Sin embargo, la depreciación del yen está dando efectos limitados en el aumento de las exportaciones. Por el lado de la finanzas públicas, en Japón donde la deuda pública asciende al 220 por ciento de su PBI, la mitad de los ingresos provenientes de la emisión de deuda nueva se utilizó para cubrir el servicio de dicha deuda. El riesgo que podrís aflorar es que los mercados financieros exigieran una mayor rentabilidad, lo que se agravaría aún más si la cuenta corriente de la balanza de pagos se mantiene en déficit por un periodo prolongado.

6. En la evolución de la economía norteamericana fue un factor principal la inclemencia del tiempo, debido a ello, el impulso del crecimiento de mediados de 2013 se desaceleró en el primer trimestre de 2014. Sin embargo, se estima que en el futuro 
se acelere nuevamente. Para ello, deberá aumentar el consumo y la inversión a un ritmo más pronunciado que en los dos últimos años, conjuntamente con una mejora del mercado laboral y del sector de la vivienda. En las previsiones económicas se asume que la política monetaria continuará siendo relajada durante el $2014 \mathrm{e}$ inicios del año siguiente. Las condiciones externas para la economía norteamericana tendrán que mejorar debido a que se prevé la demanda externa de los principales socios de los estadounidenses mejore levemente. Sin embargo, para el mundo es un importante riesgo la reducción gradual del programa de expansión cuantitativa de la Fed prevista para finales de 2014 y el aumento de las tasas de interés oficiales que se produciría a mediados de 2015 , que podrían generar una fuerte corrección de las tasas de interés a largo plazo en todo el mundo, además de una venta masiva de valores, salidas de capital de las economías en desarrollo, un incremento del costo de financiación externa y una nueva depreciación de las monedas en dichos países.

7. La vulnerabilidad de las economía en desarrollo son debido a su alta dependencia de las economías desarrolladas y en especial, de los Estados Unidos, así recientemente ante la posibilidad de que la Fed pusiera fin a su programa de expansión monetaria y de que las tasas de interés subieran provocó salidas de capitales y una fuerte depreciación de las monedas de las economías en desarrollo. Estos recientes episodios de inestabilidad observada en los mercados financieros de las economías en desarrollo suscitan el temor que estas economías se vean afectadas por un inminente ciclo de res- tricción monetaria en las principales economías desarrolladas. Un ejemplo de ello, fue la repentina y masiva salida de capitales de las economías en desarrollo que se observó a mediados de 2013 y nuevamente en enero de 2014, que provocó el desplome de los precios de los activos y las monedas.

\section{REFERENCIAS BIBLIOGRÁFICAS}

1. CEPAL (2014) Balance Preliminar de las Economías de América Latina y el Caribe 2013, Santiago de Chile.

2. CEPAL (2013) Balance Preliminar de las Economías de América Latina y el Caribe 2012, Santiago de Chile.

3. CEPAL (2014) Perspectivas Económicas de América Latina 2013, Santiago de Chile.

4. Consejo Económico y Social, Situación y Perspectivas de la Economía Mundial 2014, Naciones Unidas, Nueva York.

5. FMI (2013) Perspectivas de la Economía Mundial, Informe WEO, Washington D. C.

6. OIT (2014) Tendencias Mundiales del Empleo 2014, Ginebra.

7. OMC (2014) Informe sobre el Comercio Mundial 2013, Ginebra.

8. UN/DESA (2014) World Economic Situation and Prospects, Update as of mid-2014, New York.

9. UN/DESA (2014) World Economic Situation and Prospects 2014, New York.

10. UN/DESA (2013) World Economic Situation and Prospects 2013, New York. 